\title{
SPREADING MECHANISMS OF COOPERATION FOR THE EVOLUTIONARY PRISONER'S DILEMMA GAMES
}

\author{
GYÖRGY SZABÓ \\ Research Institute for Technical Physics and Materials Science \\ POB. 49, H-1525 Budapest, Hungary \\ E-mail:szabo@mfa.kfki.hu
}

\begin{abstract}
We survey several mechanisms supporting the maintenance of cooperation for evolutionary Prisoner's Dilemma games. In these models players are located on the sites of a lattice or graph and they can follow one of the pure strategies: cooperation $(\mathrm{C})$ or defection (D). Their total income comes from Prisoner's Dilemma games with their neighbors. We discuss the consequences of different evolutionary rules determining the time-dependence of the strategy distribution and compare the results of spreading mechanisms of cooperation.
\end{abstract}

1. Introduction. Evolutionary Prisoner's Dilemma games are widely used to describe the emergence of cooperative behavior among selfish individuals (for reviews see [1-6] and further references therein). In the original Prisoner's Dilemma game two players should simultaneously choose between two options: defection (D) and cooperation (C) [7,8]. The selfish players wish to maximize their own income in the knowledge of payoffs dependent on their choices. The curiosity of Prisoner's Dilemma game comes from the fact that the choice of defection yields a higher income independent of the partner's choice. However, if both rational players choose defection then their individual income is lower than those obtained for mutual cooperation when the maximum total payoff is shared equally. The rational players cannot resolve this dilemma although we find many examples in the nature where mutual cooperation (e.g., altruism and ethical norms) emerges among the selfish individuals [9].

In the last decades several mechanisms (e.g., kin selection [10], application of retaliating strategies [11], and voluntary participation [12]) are reported which enforce the appearance of cooperation in the societies (for a recent survey see the paper by Nowak [13]). Now we will discuss two-strategy $(C$ and $D)$ evolutionary prisoner's dilemma games with $N$ players $(N \rightarrow \infty)$ distributed on the sites of a lattice or graph. The players play

2000 Mathematics Subject Classification: Primary 91A22; Secondary 91B72.

Key words and phrases: Prisoner's Dilemma games.

The paper is in final form and no version of it will be published elsewhere. 
iterated games with their neighbors and sometime they are allowed to modify their strategy. More precisely, the players can adopt (learn) one of the neighboring strategies in a way defined by the given evolutionary rules. The success of cooperators against defectors depends on the payoffs, noises, connectivity structure, and some other details of the evolutionary rule. Now we survey those spreading mechanisms of cooperation that increase the density of cooperators and help the whole community to reach higher total income.

2. The models. The spatial versions of the evolutionary Prisoner's Dilemma games were introduced by Nowak et al. [14,15] and these cellular automata were capable to explain the maintenance of cooperation for the iterated games with a limited range of interaction if the players follow one of the two simplest strategies. For these strategies, denoted shortly as $D$ and $C$, the player chooses always defection and cooperation, respectively. We assume that each players is located on a site $x$ of a lattice or graph and their strategy is denoted by two-dimensional unit vectors, namely

$$
\mathbf{s}_{x}=D=\left(\begin{array}{l}
1 \\
0
\end{array}\right) \quad \text { or } \quad C=\left(\begin{array}{l}
0 \\
1
\end{array}\right) .
$$

The total payoff $U_{x}$ of player $x$ comes from games with the neighbors $y \in \Omega_{x}$, that is,

$$
U_{x}=\sum_{y \in \Omega_{x}} \mathbf{s}_{x}^{+} \mathbf{A} \cdot \mathbf{s}_{y},
$$

where $\mathbf{s}_{x}^{+}$denotes the transpose of the state vector $\mathbf{s}_{x}$, the summation runs over sites of the neighborhood $\Omega_{x}$. In the present discussion the self-interaction is excluded and the rescaled payoff matrix

$$
\mathbf{A}=\left(\begin{array}{ll}
0 & b \\
c & 1
\end{array}\right), \quad 1<b<2-c, \quad c<0 .
$$

will be considered in the limit $c \rightarrow-0[14,15]$. For these symmetric two-person games both interacting players receive 1 if they follow the $C$ strategy. Conversely, each player receives 0 if both choose $D$. The highest payoff $b$ is achieved by the defector against the cooperator receiving nothing. The parameter $b$ describes the strength of temptation to choose defection. Notice, that the total payoff $(b)$ for the defector-cooperator co-players is less than those received by two cooperators.

In the spatial evolutionary games the equivalent players play repeated games with their neighbors and they can modify their own strategy between games with neighbors. For the non-spatial models the players are located on the sites $x$ of a graph (network) where the edges define the connected players (neighbors) and the degree $z_{x}$ gives the number of neighbors for the player residing at site $x$.

For most of the evolutionary games the players wish to maximize their own total payoff by adopting one of the more successful strategies available in their neighborhood. This type of dynamics is analogous to the Darwinian selection in the ecological systems [16,17]. There are different ways (algorithms) defining how the players choose a new strategy. In this work our discussion will be restricted to two strategy update mechanisms, namely, synchronized update and random sequential pairwise comparison. 
2.1. Synchronized update. This evolutionary rule was suggested and studied by Nowak et al. $[14,15]$ and by many others (see e.g., [18]) during the subsequent years.

For the synchronized update the system is analogous to a cellular automaton where the strategy distribution $\left\{\mathbf{s}_{x}(t)\right\}$ is given in discrete time steps, i.e., $t=0,1,2, \ldots$ In the subsequent time step the new strategy distribution $\left\{\mathbf{s}_{x}(t+1)\right\}$ is determined by the payoff distribution $U_{x}(t)$ that can be evaluated by using Eq. (2). According to the rule suggested by Nowak and May [14], at time $t+1$ the player $x$ will follow the strategy of the neighboring player having the highest total payoff at time $t$. The generalization of this rule to arbitrary networks is straightforward and the results are discussed in papers [19-22].

2.2. Random sequential pairwise comparison. In this case the players refresh their strategy individually by repeating the subsequent elementary steps. We choose two neighboring players at the sites $x$ and $y$ at random and we evaluate their payoff $\left(U_{x}\right.$ and $\left.U_{y}\right)$ given by Eq. (2). Player $x$ adopts the neighboring strategy $\mathbf{s}_{y}$ with a probability depending on the payoff difference

$$
W\left[\mathbf{s}_{x} \rightarrow \mathbf{s}_{y}\right]=\frac{1}{1+\exp \left[\left(U_{x}-U_{y}\right) / K\right]},
$$

where $K$ (called temperature) characterizes the magnitude of noise [23-26]. It is worth mentioning that this evolutionary rule allows irrational choices, that is the players can adopt the less successful strategies with a low probability.

The above strategy adoption (learning) rules do not change the homogeneous states $\left(e . g ., s_{x}=D\right)$. These spatial distributions are called absorbing states and whenever one of the homogeneous states is reached the system remains there forever. During Monte Carlo simulations this phenomenon can be observed frequently for small system sizes after a transient time whose average value increases very fast with $N$. For large systems the inside of homogeneous regions remains unchanged because variation can occur only for those sites where one of the neighbors follows the opposite strategy.

Henceforth we assume that the system is started from a random initial state for sufficiently large sizes to avoid the mentioned small size effect. When repeating the described elementary steps the system evolves towards a state which can be characterized by the average density $\rho$ of cooperators. Before considering the numerical results for different neighborhood structures we briefly survey the prediction of mean-field theory.

3. Prediction of mean-field theory. Within the framework of mean-field theory the system is characterized by the average density $\rho$ of cooperators. This means that the average number of cooperators in the neighborhood of a given player is $z \rho$ if each site has $z$ neighbors. Consequently, the average payoff for $C$ and $D$ strategies are

$$
U_{C}=z \rho \text { and } U_{D}=z \rho b .
$$

Assuming that the strategy adoption takes place between two randomly chosen players following $D$ and $C$ strategy then the dynamical rule with random sequential pairwise comparison [defined by Eq. (4)] yields the following equation of motion for the concen- 
tration of cooperators:

$$
\begin{aligned}
\frac{\partial \rho}{\partial t} & =\rho(1-\rho)[W(D \rightarrow C)-W(C \rightarrow D)] \\
& =-\rho(1-\rho) \tanh \left(\frac{U_{D}-U_{C}}{2 K}\right) .
\end{aligned}
$$

In this system $\rho$ tends to zero as $t \rightarrow \infty$ since $U_{D}>U_{C}$ independently of the initial state and the value of $z$ and $K$. Consequently, the cooperators die out for those evolutionary Prisoner's Dilemma games where the conditions of mean-field approximation are satisfied. Here it is worth mentioning that $\rho$ tends to 1 for $b<1$ representing the so-called stag-hunt game [5-8].

It is emphasized that the conditions of mean-field theory are satisfied for infinite range of interaction in a large system $(z=N-1)$, or even in a spatial model where the player's distribution is well mixed before they play game with each other.

According to the mean-field theory significant reduction is expected in $\rho$ if the spatial distribution of $C$ and $D$ strategies is random (uncorrelated). This phenomenon can be observed in many models after starting the system from a random initial state (see below).

4. Spreading of cooperation for synchronized strategy update. Now we consider a cellular automaton type system where the players are distributed on a square lattice and their income comes from Prisoner's Dilemma games with their first- and second neighbors $(z=8)$. The strategies are refreshed simultaneously at times $t=1,2, \ldots$ in such a way [14] that each player adopts the best strategy available in her neighborhood.
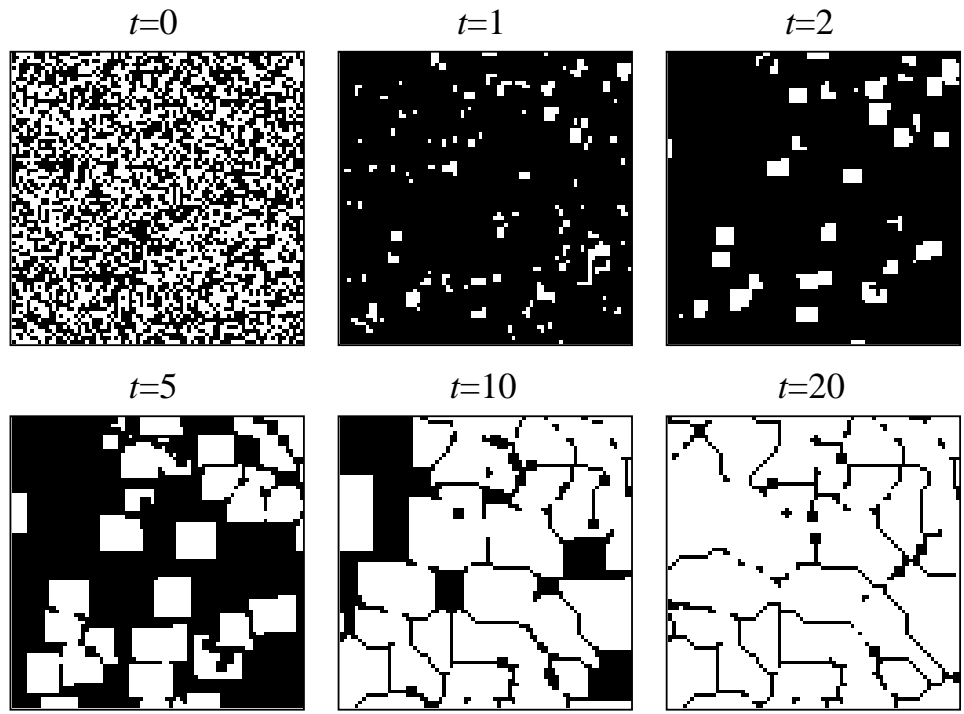

Fig. 1. Evolution of the spatial distribution of $\mathrm{C}$ (white) and D (black) strategies on a square lattice for a cellular automaton rule based on the adoption of the best strategies from the nearest and next-nearest neighbors in discrete time steps $t$ if the system is started from a random initial state at $b=1.31$. 
In order to demonstrate the time evolution of the spatial distribution of strategies Figure 1 shows six consecutive snapshots on an $80 \times 80$ portion of a larger system with periodic boundary conditions. The system is started $(t=0)$ from a random distribution of $D$ and $C$ strategies with $\rho(t=0)=0.5$. In agreement with the prediction of meanfield theory a sudden reduction of $\rho$ can be observed after the first step. In fact, only the sufficiently large rectangular blocks of cooperators could remain alive against the invasion of defectors. In the next steps the rectangular blocks of cooperators grow linearly with time because along the horizontal and vertical boundaries (separating the domains of $D$ and $C$ strategies) $C$ invasions occur due to the support received by $C$ s from their background. Quantitatively, along the horizontal and vertical boundaries the cooperators receive $U_{C}=5$ meanwhile the defectors' payoff is $U_{D}=3 b$. The cooperator invasion is stopped when two fronts meet, more precisely, when the regions of cooperators are separated by defectors who can exploit two cooperators at least. This is the way how the spatial distribution of strategies evolves towards a "frozen" pattern where the large regions of cooperators are separated by (one-site thick) chains of defectors. The final pattern depends on the initial state and the value of $b$ (for details see refs. $[14,15]$ ). In general, the average density of cooperators is determined by a dynamical balance between the cooperator invasions and defector invasions. Defector invasions occur along the irregular fronts. On the contrary, cooperator invasions can be maintained step by step along the (horizontal and vertical) straight line fronts due to the simultaneous strategy refreshment. This latter process is forbidden for random sequential updates providing a less efficient way to maintain the cooperative behavior.

\section{Spreading of cooperators for random sequential pairwise comparison. First} we consider the spreading of cooperators on a kagome lattice $(z=4)$ when the strategy adoption mechanism is defined by the random sequential pairwise comparison described above (see subsection 2.2). One can easily check that the cooperative behavior cannot spread away from a single cooperator surrounded by defectors in the zero temperature limit $(K \rightarrow 0)$. As well as for synchronized strategy updates, the adoption of cooperator strategy can become attractive if the $C$ players form compact colonies. On the kagome lattice three cooperators forming a triangle (see Figure 2) can support the spreading of cooperation in the zero temperature limit.

Figure 2 shows the possible variations in the spatial distribution of strategies for consecutive steps if initially three (neighboring) cooperators are located in the sea of defectors. In this situation the highest income is received by the three cooperators $\left(U_{C}=2\right)$ meanwhile the neighboring defectors have a lower income $\left(U_{D}^{(n)}=b\right)$ and the rest of defectors receive nothing $\left(U_{D}^{(r)}=0\right)$. In the zero temperature limit the only possible event is that one of the neighboring defectors adopts the cooperator strategy. The total income of this new cooperative player, however, is decreased $\left(U_{C}^{(n n)}=1\right)$ and becomes less than those of her neighboring defectors. Consequently, the most likely strategy change is that this player turns back to defection again and a similar temporary cooperation can occur several times in the neighborhood of the cooperator triplet. The new-born cooperator strategy can be fixed if her most successful neighboring defector 


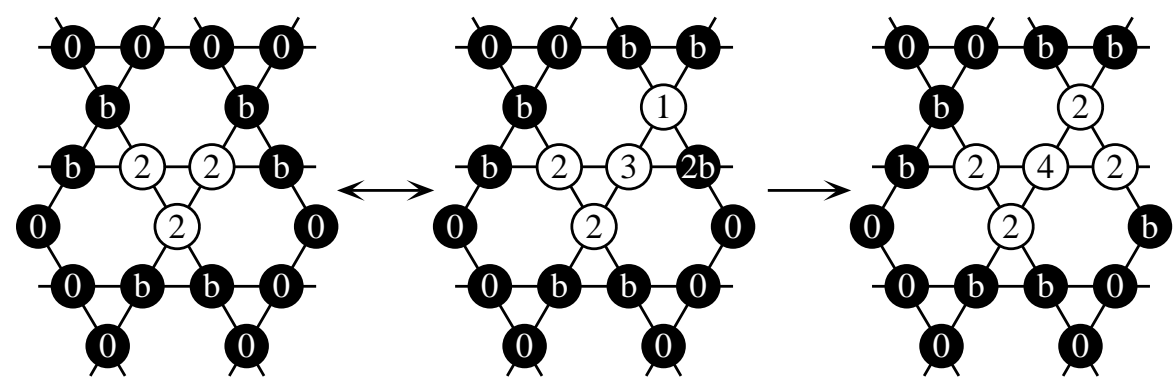

Fig. 2. Appearance of a new cooperator triplet (white bullets) in the sea of defectors (black bullets) for the random sequential pairwise comparison rule (in the limit $K \rightarrow 0$ ) if initially only one cooperator triplet exists on the kagome lattice. Figures in bullets refer to payoff dependent on the neighboring strategies. The arrows indicate the direction of possible transitions.

$\left(U_{D}^{m s}=2 b\right)$ has an opportunity to adopt strategy from the most successful cooperator $\left(U_{C}^{m s}=3\right)$. After these two consecutive steps two stable cooperator triplets (with a common player) occur and the iteration of this process yields a growing tree of cooperator triplets. The growth of the tree of triangles can be stopped by defectors separating two branches of trees formed by cooperator triplets.

For the present structure the above spreading mechanism of cooperation occurs if the temptation to choose defection does not exceed a critical value, that is, $b<b_{c}(K=0)=$ $3 / 2$ in the limit $K \rightarrow 0$. For finite temperatures the efficiency of this spreading process is decreased by the irrational strategy adoptions. As a result, $\rho$ decreases monotonically when $b$ is increased for fixed temperature $K$ as demonstrated in Figure 3.

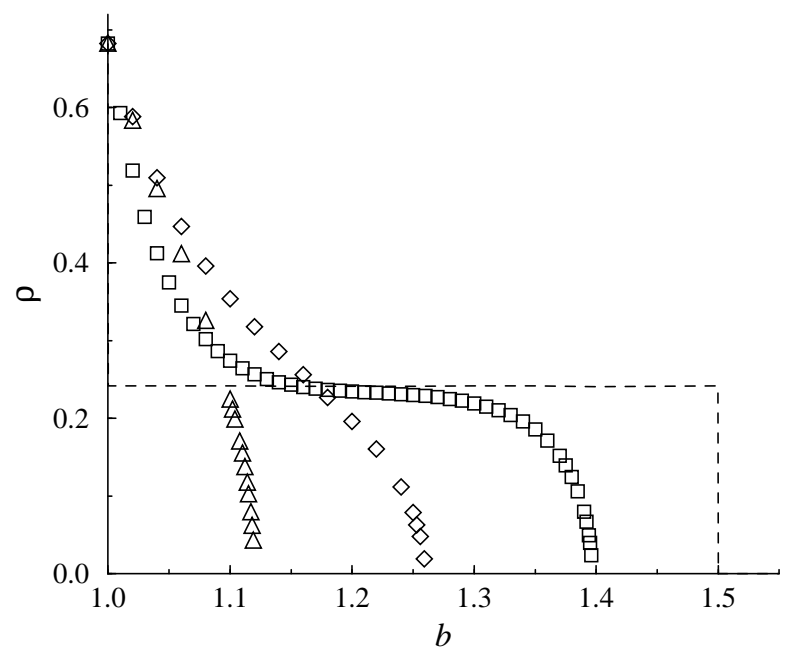

Fig. 3. Monte Carlo data for the average $\rho$ density of cooperators versus $b$ on the kagome lattice at three different temperatures: $K=0.1$ (squares); 0.2 (diamonds); 0.5 (triangles). The zero temperature limit is illustrated by the dashed line. 


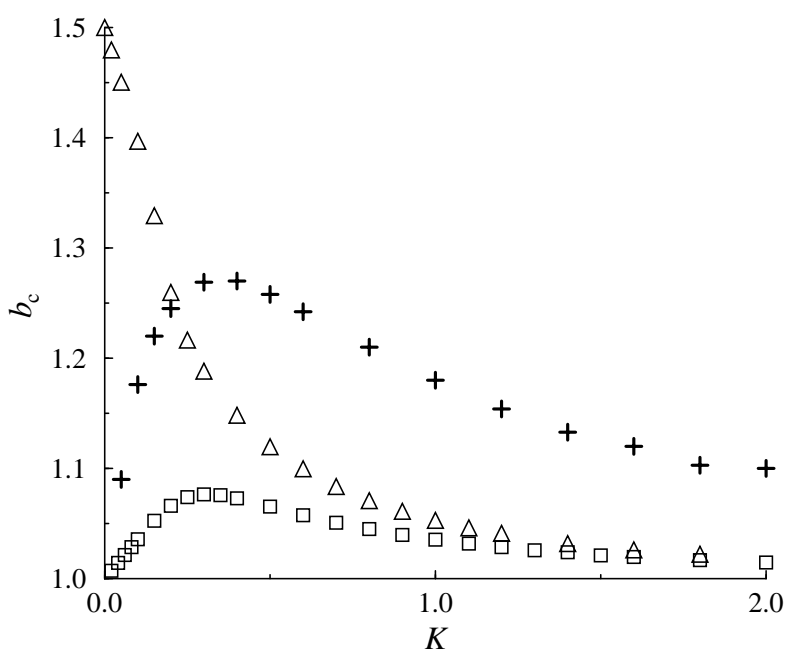

Fig. 4. The critical value of $b$ as a function of $K$ when using pairwise comparison for three different lattices where each site has four neighbors (triangles: kagome lattice; squares: square lattice; pluses: Bethe lattice).

The cooperative strategies can be maintained (i.e., $\rho>0)$ for $b<b_{c}(K)$ where $b_{c}(K)$ is a monotonously decreasing function of $K$ as illustrated by triangles in Figure 4. Using Monte Carlo simulations similar behavior is found for many other $d$-dimensional $(d>1)$ lattices (e.g., two-dimensional square lattice with first- and second neighbor interactions $(z=8)$, triangular lattice $(z=6)$, and body centered cubic lattice $(z=8))$ which can be spanned by overlapping triangles [27]. It is conjectured that overlapping triangles in the connectivity structure can support the spreading of cooperators although for most of the investigated structures the overlapping triangles can have two common lattice sites that makes the spreading mechanism of cooperation more complicated. Only one exception is found until now that does not satisfy this conjecture, namely, the one-dimensional lattice with first- and second neighbor interactions $(z=4)$ whose behavior is strongly affected by its one-dimensional character. The rigorous investigation of the evolutionary Prisoner's Dilemma game on this structure is in progress.

Significantly different behavior is found by Monte Carlo simulations when considering the same model on the square and Bethe lattices for $z=4$ as illustrated in Figure 4 . For both cases $b_{c}(K)$ has a local maximum for an optimum temperature and $b_{c}(K)$ tends to 1 if $K \rightarrow 0$. These structures do not support the spreading of $C$ strategies if $K \rightarrow 0$ as demonstrated in Figure 5.

Let us consider what happens if we have four cooperators forming a square $(2 \times 2)$ block in the sea of defectors on the square lattice. For such an arrangement the cooperators have the highest income $\left(U_{C}=2\right)$, the neighboring defectors can exploit only one cooperator (i.e., $U_{D}^{(n 1)}=b$ ), and all the other defectors receive nothing. Consequently, the cooperators will not adopt the defector strategy in the low temperature limit. At the same time one of the neighboring defectors can easily adopt the $C$ strategy and this event will be so beneficial to one of the neighboring defectors (receiving $2 b$ ) that her strategy can 


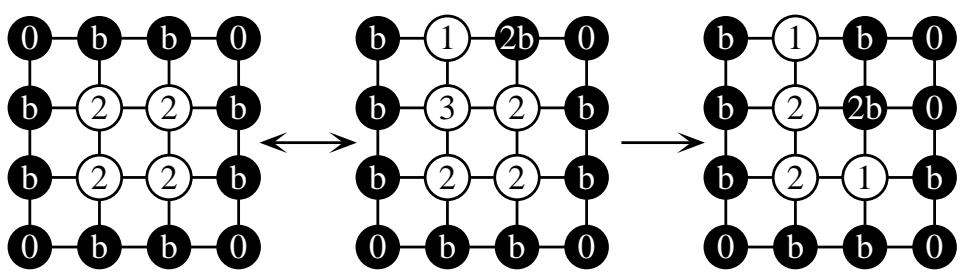

Fig. 5. Relevant consecutive configurations during the destruction of a $2 \times 2$ block of cooperators (located in the sea of defectors) when the strategy adoption is governed by random pairwise comparison on the square lattice.

be adopted by both of her neighboring $C$ players in the subsequent strategy adoption process. Consequently, the stable block of four cooperators will be destroyed because the rest of cooperators are not capable to survive in the zero temperature limit.

The (isolated) closed loops of cooperators remain stable configurations in those structures where the neighboring defectors are capable to exploit only one cooperator if the irrational strategy adoption $\left(U_{x}>U_{y}\right)$ is forbidden. The evolutionary rule defined by Eq. (4), however, allows irrational choices (if $K>0$ ) which are capable to destroy these loops. After adopting a defector within the loops of cooperators the system behavior becomes analogous to a one dimensional problem with $z=2$ where the cooperator sites are invaded by defectors with a constant average velocity. Consequently, in the absence of an effective reconstruction mechanism (e.g., a cooperation spreading process described above) the isolated loops of cooperators vanish exponentially with a typical relaxation time proportional to $\exp [(2-b) / K]$. This feature causes serious difficulties when considering these systems by Monte Carlo simulations for sufficiently low temperatures.

The appearance of cooperators through noise effects is supported for those constellations where the $C$ strategies form colonies because within these colonies the cooperators help each other. In general, the probability of a $C-C$ pair is higher than $\rho^{2}$ predicted by the mean-field theory. This fact is due to the present evolutionary rule favoring the appearance of $C-C$ and $D-D$ pairs that gives an extra advantage to cooperators against defectors. For large temperatures, however, this type of correlation is reduced and the system behavior can be well approximated by a mean-field theory predicting $b_{c}=1$. Figure 4 shows that for the spatial models $b_{c}(K) \rightarrow 1$ if $K$ goes to infinity. Here we have to emphasize that the high temperature limit is analogous to the so-called weak selection limit used in the biological literature, e.g., [28-30].

In agreement with the expectations the mean-field type behavior is enforced when increasing the number of neighbors independently of the evolutionary rules [14,15,31-35]. In order to check this tendency for the pairwise comparison, the above investigations were repeated on many other lattice structures characterized by $z=4,6$ and 8 . According to the preliminary numerical results the highest critical value of $b_{c}$ (namely, $b_{c}(K=0)=$ $3 / 2$ ) is found on the kagome lattice in the limit $K \rightarrow 0$.

The existence of an optimum temperature in the maintenance of cooperation is accompanied by a proper temperature-dependence in the density of cooperators for a fixed 


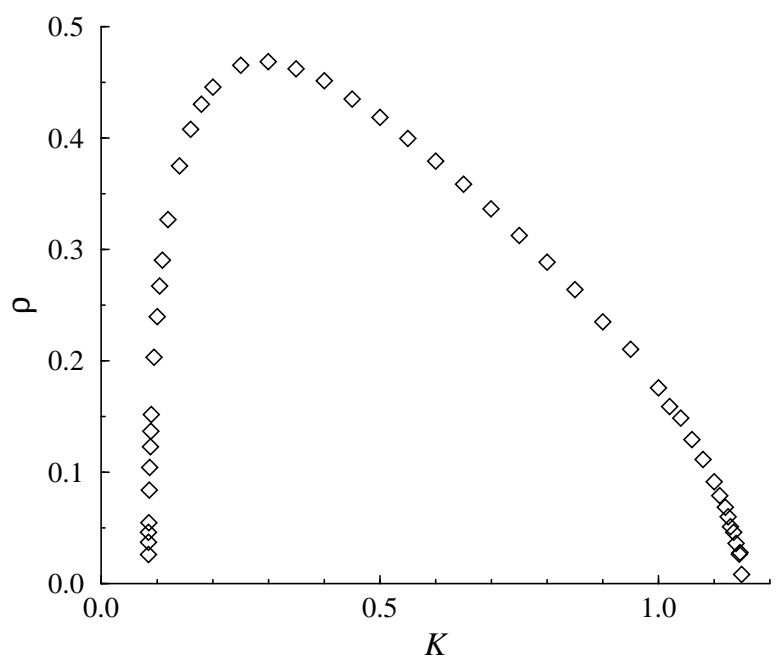

Fig. 6. Average density of cooperators as a function of temperature $K$ on the square lattice for $b=1.03$.

$b$ value as shown in Figure 6 [26]. This behavior resembles the coherence resonance [36-38] that was already predicted for some evolutionary games [39] and many other noise driven excitable systems [40].

The extinction process of cooperators at the edges of coexistence region $\left(K=K_{c 1}\right.$ and $K_{c 2}$ ) exhibits the features of a critical transition belonging to the directed percolation universality class $[25,41]$. In other words, $\rho$ vanishes as

$$
\rho \simeq\left|K-K_{c}\right|^{\beta} \text {. }
$$

where $K_{c}=K_{c 1}(b)$ or $K_{c 2}(b)$ and $\beta=0.58(1)$ for the two-dimensional systems. The algebraic behavior of $\rho$ is accompanied with a power law divergency in the density fluctuation, correlation length, and relaxation time. This universal behavior is related to the existence of an absorbing state (for details see [42]). As mentioned above the present system has two homogeneous absorbing states, therefore the extinction of defectors (appearing for suitable values of parameters) has similar features $[6,25,41]$.

The relevance of the above mentioned topological features is confirmed by the generalized versions of mean-field (or pair) approximations when we determine the probability of each possible configuration on a set of small clusters formed by $n$ lattice sites (for technical details see [6]). Evidently, this method becomes more and more reliable if we choose a larger $n$. As mentioned above, when we increase the value of $b$ the traditional mean-field theory $(n=1)$ predicts a sudden change from the homogeneous $D$ state to the homogeneous $C$ state at $b=b_{c}^{m f}(K)=1$ independently of the temperature. The pair approximation $(n=2)$ is already capable to describe qualitatively the coexistence of $C$ and $D$ strategies, however, the numerical results show poor agreement with the Monte Carlo data, particularly at the zero temperature limit where its prediction becomes qualitatively wrong $\left(b_{c}^{(p a)}(K) \rightarrow 2\right.$ if $\left.K \rightarrow 0\right)$. The three- and five-site approximations on 
the kagome lattice and also the four- and nine-site approximations on the square lattices could reproduce the Monte Carlo data with an adequate accuracy [25] because these clusters of sites take into account the loops in the connectivity structures.

There is another way to demonstrate the importance of small (entangled) loops in the connectivity structures. Namely, the evolutionary prisoner's dilemma game can also be studied on the (loop-free) Bethe lattice with using the generalized versions of mean-field theories. Similar Monte Carlo results are expected on a sufficiently large random regular graph that becomes locally similar to the Bethe lattice because the typical (minimal) loop size is proportional to $\ln N$ [42]. In Figure 4 the plus symbols represent the Monte Carlo data obtained on a random regular graph of degree $z=4$ if we use the same evolutionary rules for $N=10^{6}$. To reproduce this behavior with the generalized mean-field theory we had to extend this analysis to the level of eleven-site clusters. The $K$-dependence of the critical value of temptation $\left(b_{c}^{(r r g)}(K)\right)$ on the random regular graph is qualitatively similar to those found on the square lattice, although $b_{c}^{(r r g)}(K)$ is significantly larger than $b_{c}^{(s l)}(K)$ for arbitrary temperatures. The high temperature (weak selection) behavior of a similar evolutionary prisoner's dilemma game was analyzed by Ohtsuki et al. [28] on finite graphs, and their results allow the coexistence of $C$ and $D$ strategies within a range of $b$ (if $K \rightarrow \infty$ ). It is hoped that the asymptotic behavior in these types of models will be clarified within a few years.

One of the most relevant messages of the numerical data plotted in Figure 9 is that the kagome lattice provides the best lattice connectivity structure to achieve the highest critical $b_{c}$ value for low temperatures. For the opposite case $(K \rightarrow \infty)$, the loop-free structures can help the maintenance of cooperative behavior most efficiently if the number of neighbors (degree) is fixed (here $z=4$ ). In the subsequent section we will consider the effect of inhomogeneous degree distribution on the density of cooperators if the connectivity structure is defined by the so-called scale-free networks.

6. Enhancement of cooperation on scale-free networks. In this section we discuss briefly the results of Santos et al. [44,45] who considered two-strategy prisoner's dilemma games with several evolutionary rules on a random network suggested by Barabási and Albert [46]. One of the applied strategy adoption rule was similar to the mentioned pairwise comparison at a low temperature. It is reported that the average density of cooperators was surprisingly large $(\rho>0.8)$ in the whole region of temptation $(1<b<2)$ and the relevant results are not affected significantly by the details of evolutionary rules.

The creation of the Barabási-Albert random network can start from three connected sites and it is grown by adding a new site to the system in each elementary step. The new sites are linked to two existing sites chosen randomly with probabilities proportional to the actual degree (further details in [46]). After $(N-3)$ elementary steps the resultant graph consist of $N$ sites which can have different number of neighbors $(z)$ in such a way that the probability to find $z$ neighbors at a given sites is proportional to $f(z) \sim 1 / z^{3}$ if $z$ does not exceed a natural limit dependent on $N$. During the growth process the average number of neighbors tends to $\langle z\rangle=4$. This connectivity structure contains sites (called hubs) that has a large number of neighbors. In general, the large hubs are created at 

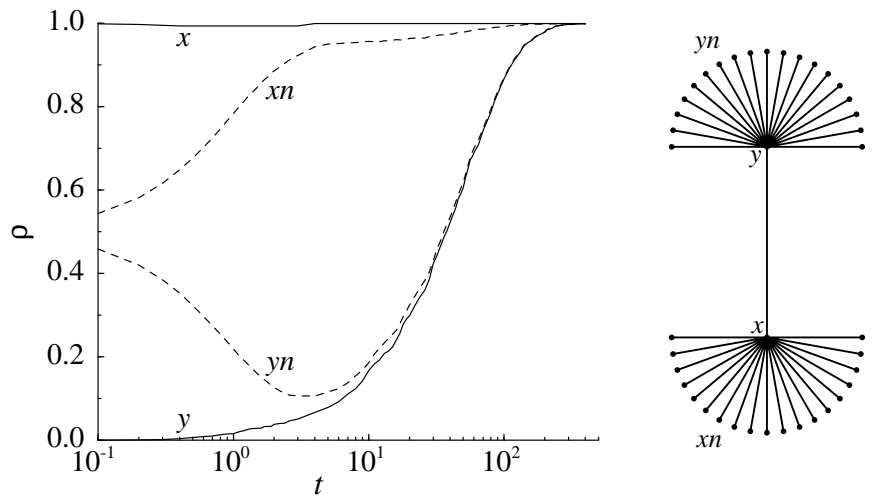

Fig. 7. Time-dependence of the frequency of cooperators on the sites of cooperator $(x)$ and defector $(y)$ hubs and on their neighborhood ( $x n$ and $y n)$. The plotted data are averaged over 1000 runs for $R=0.5$ characterizing the effects of neglected links and noise.

the beginning of network creation and these sites are typically linked to other hub(s). This latter feature plays a crucial role in the mechanism supporting the spreading of cooperation as described by Santos et al. [44,45]. Now we will discuss this mechanism throughout a numerical example.

Let us consider a small portion of players residing on two connected hubs and their neighboring sites as illustrated schematically in the right hand plot of Figure 7 . For sake of simplicity we assume that the connected hubs (denoted by $x$ and $y$ ) have $N_{x n}=$ $N_{y n}=49$ neighbors whose further links are not denoted. Thus the whole subsystem has $N=2+N_{x n}+N_{y n}=100$ sites. It is also assumed that the number of neighbors at the neighboring sites ( $x n$ and $y n$ ) is significantly less than 50 . To demonstrate the competition between the cooperator and defector hubs the system is started from an initial state where $\mathbf{s}_{x}(t=0)=C$ and $\mathbf{s}_{y}(t=0)=D$, and their neighborhood follows the $D$ and $C$ strategies with the same probability, that is, $\rho_{x n}(t=0)=\rho_{y n}(t=0)=0.5$. In the model suggested by Santos et al. the evolutionary rule is controlled by the difference of total payoffs favoring hubs. Consequently, the strategy adoption process can be simplified in the subsystem.

During the strategy adoption process on this subsystem we choose randomly a player to adopt strategy from one of her neighbors selected also at random. If one of the hubs ( $x$ or $y$ ) has a chance to adopt strategy from the neighbors of low degree ( $x n$ or $y n$ ) then nothing happens because generally $U_{x}>>U_{x n}$ and $U_{y}>>U_{y n}$. In the opposite case, the $x n$ and $y n$ players adopt the current strategy of players $x$ and $y$, respectively. The effect of the neglected interactions and learning connections are taken into consideration by allowing the players $x n$ and $y n$ to adopt a strategy with a probability $R$ from a player selected randomly in the whole subsystem. The results of numerical investigations are shown in Figure 7.

Figure 7 illustrates what happens if the connected hubs follow opposite strategies at the beginning. Initially, the total income of the cooperator hub is lower then those received by the defector hub because they practically have an equal number of coopera- 
tive co-players. However, each hub transfers her strategy to her neighborhood and these changes in their neighborhood are beneficial to cooperator hubs and disadvantageous to defector hubs. Consequently, after a short transient time the cooperator hubs become the best players whose strategy is adopted by all the other (linked) players. This phenomenon is robust if the degree of hubs exceeds significantly the maximum number of neighbors for their co-players. The robustness of this mechanism can be confirmed by the present numerical example within a wide range of parameters $\left(N_{x}, N_{y}, \mathrm{R}\right.$ and b). Further systematic investigations are required to clarify the effect of noise (temperature) and degree distribution on the average density of cooperators in the large $N$ limit.

In the original models considered by Santos et al. the simulations were performed for different values of $N$. For small sizes the result of simulation depends on the graph and initial strategy distribution. As a result, the state of the isolated defector hub(s) can remain practically unchanged during the simulations. A similar phenomenon is reported by Gómez-Gardañes et al. [47] who have considered the same model with a synchronized strategy update. Santos et al. [44,45] have checked the consequence of the removal of links connecting large hubs. In agreement with the expectation, the numerical investigation indicated a reduction in the average density of cooperators.

The additional effect of cooperation spreading mediated by the overlapping triangles on the density of cooperators was also investigated by Santos et al. when considering the model on another scale-free network suggested by Dorogovtsev et al. [48]. In this case the network creation is slightly modified: the additional new sites are linked to both ends of an edge selected at random. This creation procedure yields a network that can be spanned by (one-site) overlapping triangles meanwhile the degree distribution remains practically unchanged. On this network the simulations indicated a slight increase in $\rho$ in comparison to results obtained on the Barabási-Albert network.

The topological conditions of the mentioned mechanism are well satisfied for the scale-free networks as well as for many other connectivity structure exhibiting connected large hubs. There exist, however, many other non-regular (social) networks where the inhomogeneity in the degree distribution is not so relevant (e.g., Erdôs-Rényi graphs [49] and Watts-Strogatz small-world structure [50]). The numerical investigations of the evolutionary prisoner's dilemma games on different non-regular structures have demonstrated some improvement in the cooperative behavior related to the inhomogeneity in the number of neighbors [21,51-55].

The above models on non-regular networks involve intrinsically an inhomogeneous strategy adoption probability due to the entanglement between connectivity structure and dynamical rules [52,53]. Namely, the strategy adoption becomes asymmetric between two neighbors if they have a different number of neighbors when using the above described evolutionary rules. In the following section we discuss particular cases where the spatial network is regular while the dynamical rule is inhomogeneous.

7. Enhancement of cooperation for inhomogeneous dynamics. Remarkable increase of cooperation is observed for several systems where the inhomogeneous imitation activity is built into the models artificially to characterize the asymmetric and different 
influence of players to each other $[51,55,56]$. For example, Wu et al. $[55,56]$ have considered a model where the influence of player $y$ on her neighbor $x$ is quantified by a random parameter $w_{x y}\left(0<w_{x y} \neq w_{y x}<1\right)$ affecting the preferential selection of a neighbor whom the strategy can be adopted from.

The numerical justification of the enhancement of cooperation will be demonstrated for one of the simplified versions of the model [57] suggested originally by Wu et al. $[55,56]$. For sake of simplicity we assume that $w_{x y}$ is described by one of the two possible values in such a way that the spatial distribution $w_{x y}$ is quenched during the simulations. Now our investigation will be focused on the spatial distribution of $w_{x y}$ that provides the highest enhancement of cooperation.

In the present model we have two types of players (denoted as $A$ and $B$ ) distributed randomly on a two-dimensional lattice before the start of simulation. The fixed spatial distribution of players is defined by a set of site variables $n_{x}=A$ or $B$ and characterized by densities $\nu$ and $(1-\nu)$. Independently of $n_{x}$ the players can follow one of the $s_{x}=C$ or $D$ strategies and their total payoff $U_{x}$ comes from one Prisoner's Dilemma game with the neighbors as given by the expression (2).

The type of players becomes relevant in the iterated strategy adoption processes controlling the variation of strategy distribution. More precisely, the pairwise comparison evolutionary process (detailed in Section 2.2) is modified:

$$
W\left(s_{x} \rightarrow s_{y}\right)=w_{x y} \frac{1}{1+\exp \left[\left(U_{x}-U_{y}\right) / K\right]}
$$

where temperature $K$ characterizes the uncertainties (noises) and the pre-factor $w_{x y}$ is given as

$$
w_{x y}= \begin{cases}1, & \text { if } n_{y}=A, \\ w, & \text { if } n_{y}=B,\end{cases}
$$

and the fixed value of $w(0<w<1)$ describes the strength of reduced teaching activity if the site $y$ is occupied by a player of type $B$. This means that here the players $A$ and $B$ have different teaching activity (or efficiency). Such a feature can occur in some communities where the young and old (or attractive and repulsive) persons have different capability to transfer their own strategy.

Figure 8 summarizes the results of Monte Carlo simulations when varying the density of $B$ players on a kagome lattice for fixed $b$ and $K$ values. In this system the stationary states obtained for $\nu=0$ and 1 are equivalent because the homogeneous reduction of strategy adoptions yields only slower evolution towards the same stationary state. The largest increase in $\rho$ can be observed if the portion of $A$ and $B$ types players are approximately equivalent. Notice that the increase of the density of cooperators $(\Delta \rho)$ varies linearly with the concentration of the minority type of players, i.e., $\Delta \rho \simeq \alpha \nu$ if $\nu<<1$ and $\Delta \rho \simeq \alpha(1-\nu)$ if $(1-\nu)<<1$ where $\alpha$ increases monotonously with $(1-w)$. The increase of the density of cooperators may be so large that defectors can die out within a range of $\nu$ as demonstrated in Figure 8 by data for $w=0.1$.

Unfortunately the clear and simple explanation of this mechanism is not yet known although similar behaviors are found on the square lattice, too. For both two-dimensional 


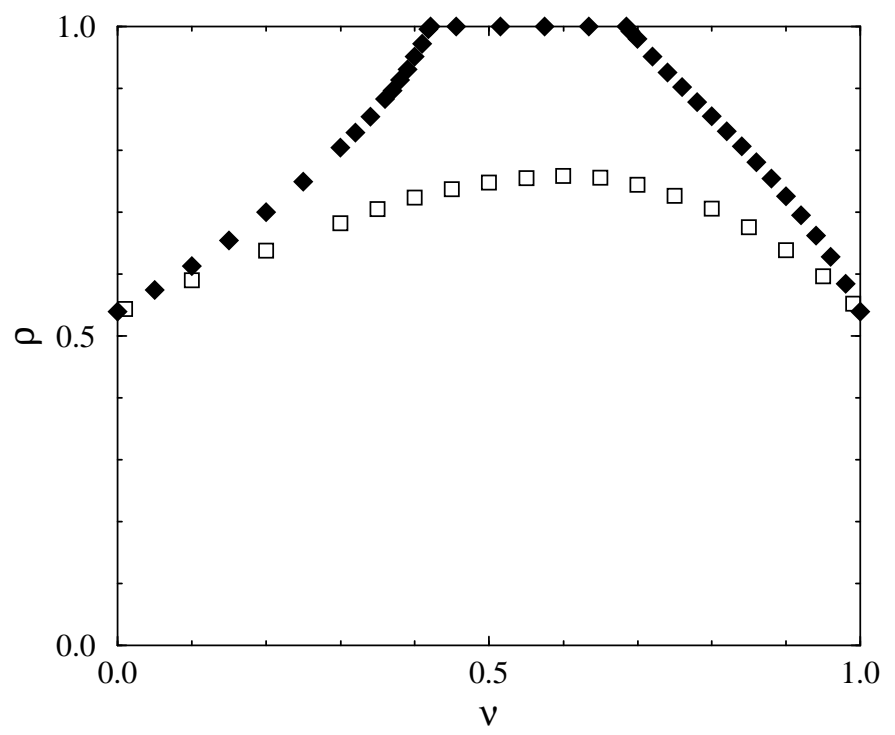

Fig. 8. Density $\rho$ of cooperators as a function of $\nu$ (density of players with reduced teaching activity) for $w=0.1$ and 0.2 on the kagome lattice at $b=1.03$ and $K=0.5$.

structures the improvement vanishes in the low temperature limit and remains remarkable even if $K \rightarrow \infty$. The main features of this phenomenon are reproduced qualitatively well by the pair approximation [57].

The hidden curiosity of this mechanism becomes striking when repeating the same analysis with choosing other possibilities in the randomness of $w_{x y}$. For example, significantly smaller variations of $\rho$ are observed for those cases when the learning (instead of teaching) activity is blocked at the $B$ type players by defining the pre-factor $w_{x y}$ in Eq. (8) as

$$
w_{x y}= \begin{cases}1, & \text { if } n_{x}=A, \\ w, & \text { if } n_{x}=B .\end{cases}
$$

It is worth mentioning that the spatial distribution of $w_{x y}$ becomes equivalent for the cases defined by Eqs. (9) and (10) if the (periodical) spatial arrangement of players $A$ and $B$ is resembling the black and white squares on the chessboard. Interestingly, this type of dynamical inhomogeneity yields only a slight change in $\rho$ that may be either positive or negative depending on the model parameters.

From the mentioned numerical results one can conclude that the random distribution of influential players representing asymmetric teaching-learning activity in the community can also support the maintenance of cooperative behavior [52,55-57]. In the following section we will compare the strength of all the above mentioned mechanisms.

8. Summary. In the present work we have surveyed the spreading mechanisms of cooperation supporting the cooperative behaviors in the evolutionary prisoner's dilemma games. The discussion is restricted to systems where the players can use only two strate- 
gies $(C$ and $D)$ and the interaction between them is described by a fixed network (graph).

On the square lattice it is shown that along the horizontal and vertical fronts (separating $C$ and $D$ domains) the cooperator invasion is supported by their background until the income of exploiting defectors do not exceed a threshold value dependent on the neighborhood. The advance of $C$ players along these straight line fronts can be directly utilized by synchronized strategy update rules that maintain the invasion with a constant velocity. These type of $C$ invasion can be stopped when the front lines collide, more precisely, when the $C$ domains are separated from each other by one-site thick layers of defectors.

The noisy rules make the straight line fronts irregular that can prevent the expansion of $C$ domains. Despite it, the $C$ invasion can occur for some regular connectivity structures even if the strategy adoption is controlled by random sequential pairwise comparison in the zero temperature limit. It is demonstrated that the one-site overlapping triangles in the connectivity structure (for $z=4$ ) help the spreading of cooperation. In this case one can observe growing trees of cooperator triplets whose growth can be stopped by defectors separating two branches of the cooperator trees. In some sense this phenomenon is similar to those observed for the cellular automaton type rules and its efficiency is weaker as demonstrated in Figure 9 where the $b$-dependence of $\rho$ is compared for different connectivity structures and evolutionary rules.

The numerical and analytical investigations of the systems with random sequential update indicate that the cooperation vanishes in the zero temperature limit for those connectivity structures which can not be spanned by overlapping triangles. In these latter cases, however, there exists an optimum temperature (noise level) where cooperators can exist with a sufficiently high density as illustrated in Figure 9. This means that the irrational strategy adoptions can also help the survival of cooperators forming colonies. It is found, furthermore, that the loop-free (non-spatial) connectivity structures provide the best regular connectivity structure for the cooperators to exist in the high temperature limit.

For the non-regular connectivity structures the connected large hubs support the emergence of cooperative behavior. The corresponding mechanism is robust on the scalefree graphs and capable to maintain cooperation with a high density $(\rho>0.8)$ in the whole region of payoff $b$ (see Figure 9). In the models suggested by Santos et al. the enhanced role of large hubs comes from the fact that the players compare their total income increasing with the number of neighbors. It is found that the efficiency of this mechanism is reduced significantly if the strategy adoption rule is based on the normalized payoff difference [e.g., $\left(U_{x} / z_{x}-U_{y} / z_{y}\right)$ where $z_{x}$ is the number of neighbors at the site $\left.x\right]$. For the non-regular connectivity structures the players are not equivalent and the symmetry between the neighboring players is broken for most of the evolutionary rules we can apply. It turns out that the asymmetry in the master-follower role between two neighboring players can also result in an improvement in the cooperative behavior.

Further systematic investigations of the discussed phenomena are in progress. The current studies can explore the interference between the mentioned mechanisms. We have 


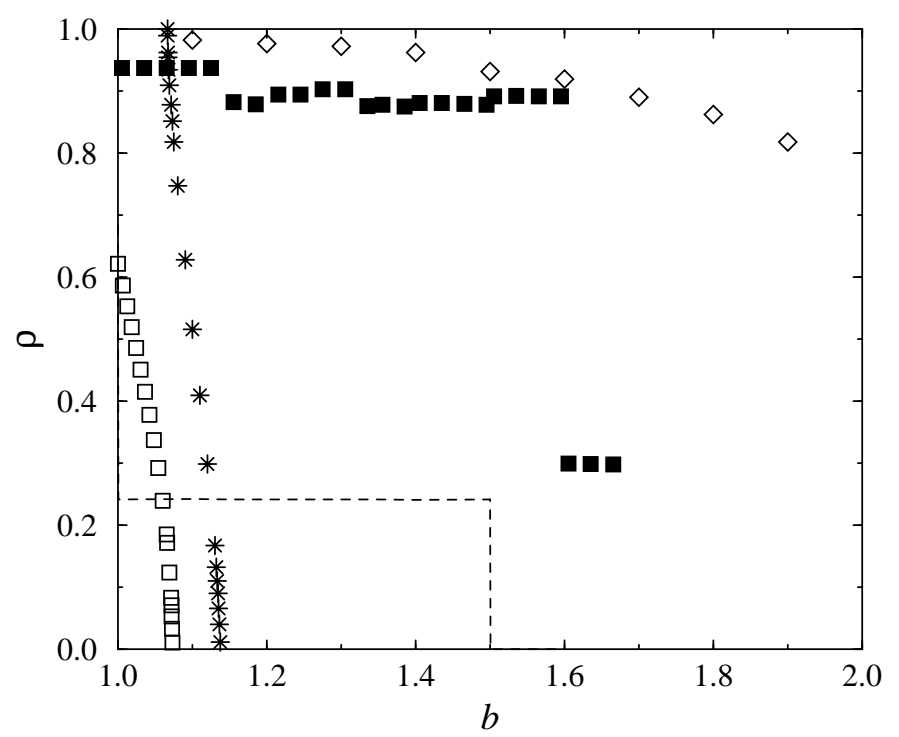

Fig. 9. Comparison of the density of cooperators as a function of $b$ for different connectivity structures. The dashed line shows the results on the kagome lattice at the zero temperature limit. Open squares represent data for the square lattice with pair-wise comparison at the optimum temperature $(K=0.4)[26]$. Diamonds denote Monte Carlo data obtained by Santos et al. [44,45] on the scale-free Barabási-Albert network. Closed squares refer to data on the square lattice with first- and second-neighbor interactions when using synchronized strategy update suggested by Nowak and May [14]. Stars refer to evolutionary game with a modified pairwise comparison where the teaching activity is blocked by a factor of $w=0.1$ for half of the players distributed randomly on a square lattice at $K=1.0$ [57].

to emphasize that recently the research of evolutionary games expands progressively. In the last years further promising results were reported including the consideration of the multilevel selection [58,59], the co-evolution of strategy distribution and connectivity structure [60,61], and the separation of learning and interaction graphs $[62,63]$.

\section{References}

[1] M. A. Nowak, Evolutionary dynamics of biological games, Science 303 (2004), 793-799.

[2] K. Lindgren and G. Nordahl, Evolutionary dynamics of spatial games, Physica D 75 (1994), 292-309.

[3] M. Nakamaru, H. Masuda and Y. Iwasa, The evolution of cooperation in a lattice-structued population, J. Theoretical Biology 184 (1997), 65-81.

[4] J. Miękisz, Statistical mechanics of spatial evolutionary games, J. Physics A: Math. Gen. 37 (2004), 9891-9906.

[5] J. Miękisz, Evolutionary game theory and population dynamics, arXiv:q-bio/0703062 (2007).

[6] G. Szabó and G. Fáth, Evolutionary games on graphs, Physics Reports (2007), in press.

[7] H. Gintis, Game Theory Evolving, Princeton University Press, Princeton, 2000. 
[8] R. Cressman, Evolutionary Dynamics and Extensive Form Games, MIT Press, Cambridge MA, 2003.

[9] M. A. Nowak and K. Sigmund, Arithmetics of mutual help, Scientific American 272 (1995), 76-81.

[10] W. Hamilton, Genetical evolution of social behavior II, J. Theoretical Biology 7 (1964), $17-52$.

[11] R. Axelrod, The Evolution of Cooperation, Basic Books, New York, 1984.

[12] C. Hauert, S. De Monte and K. Sigmund, Volunteering as Red Queen mechanism for cooperation in public goods game, Science 296 (2002), 1129-1132.

[13] M. A. Nowak, Five rules of the evolution of cooperation, Science 314 (2006), 1560-1563.

[14] M. A. Nowak and R. M. May, The spatial dilemmas of cooperation, Int. J. Bifurcation and Chaos 3 (1993), 35-78.

[15] M. A. Nowak, S. Bonhoeffer and R. M. May, More spatial games, Int. J. Bifurcation and Chaos 4 (1994), 23-56.

[16] R. Axelrod and W. D. Hamilton, The evolution of cooperation, Science 211 (1981), 13901396.

[17] J. Maynard Smith, Evolution and the Theory of Games, Cambridge University Press, Cambridge, 1982.

[18] T. Killingback and M. Doebeli, Self-organized criticality in spatial evolutionary game theory, J. Theoretical Biology 191 (1998), 335-340.

[19] G. Abramson and M. Kuperman, Social games on social network, Physical Review E 63 (2001), 030901(R).

[20] N. Masuda and K. Aihara, Spatial prisoner's dilemma optimally played in small-world networks, Physics Letters A 313 (2003), 55-61.

[21] O. Duran and R. Mulet, Evolutionary prisoner's dilemma in random graphs, Physica D 208 (2005), 257-265.

[22] J. Gómez-Gardeñes et al., Dynamical organization of cooperation in complex topologies, Physical Review Letters 98 (2007), 108103.

[23] L. E. Blume, The statistical mechanics of best-response strategy revision, Games and Economic Behavior 11 (1995), 111-145.

[24] L. E. Blume, How noise matters, Games and Economic Behavior 44 (2003), 251-271.

[25] G. Szabó and C. Tóke, Evolutionary prisoner's dilemma game on a square lattice, Physical Review E 58 (1998), 69-73.

[26] G. Szabó, J. Vukov and A. Szolnoki, Phase diagrams for an evolutionary prisoner's dilemma game on two-dimensional lattices, Physical Review E 72 (2005), 047107/1-4.

[27] J. Vukov, G. Szabó and A. Szolnoki, Cooperation in the noisy case: Prisoner's dilemma game on two types regular random graphs, Physical Review E 74 (2006), 067103/1-4.

[28] H. Ohtsuki, C. Hauert, E. Lieberman and M. A. Nowak, A simple rule for the evolution of cooperation on graphs and social networks, Nature 441 (2006), 502-505.

[29] C. Taylor, Y. Iwasa and M. A. Nowak, Symetry of fixation times in evolutionary dynamics, J. Theoretical Biology 243 (2006), 245-251.

[30] A. Traulsen, J. M. Pacheco and M. A. Nowak, Pairwise comparison and selection temperature in evolutionary game dynamics, J. Theoretical Biology 246 (2007), 522-529.

[31] M. Ifty, T. Killingback and M. Doebeli, Effect of neighborhood size and connectivity on the spatial continuous prisoner's dilemma, J. Theoretical Biology 231 (2004), 97-106.

[32] F. C. Santos, J. F. Rodrigues and J. M. Pacheco, Graph topology plays a determinant role in the evolution of cooperation, Proc. Royal Society London B 273 (2006), 51-55. 
[33] C.-L. Tang, W.-X. Wang, X. Wu and B.-H. Wang, Effects of average degree on cooperation in networked evolutionary game, Eur. Phys. J. B 53 (2006), 411-415.

[34] Z.-X. Wu and Y.-H. Wang, Cooperation enhanced by the difference between interaction and learning neighborhoods for evolutionary spatial prisoner's dilemma games, Physical Review E 75 (2007), 041114/1-7.

[35] M. H. Vainstein, A. T. C. Silva and J. J. Jeferson, Does mobility decrease cooperation?, J. Theoretical Biology 244 (2007), 722-728.

[36] M. Perc, Coherence resonance in a spatial prisoner's dilemma game, New J. Physics 8 (2006), 22 .

[37] M. Perc and M. Marhl, Evolutionary and dynamical coherence resonances in the pair approximated prisoner's dilemma game, New J. Physics 8 (2006), 142.

[38] M. Perc, Double resonance in cooperation induced by noise and network variation for an evolutionary prisoner's dilemma, New J. Physics 8 (2006), 183.

[39] A. Traulsen, T. Röhl and H. G. Schuster, Stochastic gain in population dynamics, Physical Review Letters 93 (2004), 028701.

[40] A. S. Pikovsky and J. Kurths, Coherence resonance in a noise-driven excitable system, Physical Review Letters 78 (1997), 775-778.

[41] J. N. R. Chiappin and M. J. de Oliveira, Emergence of cooperation among interacting individuals, Physical Review E 59 (1999), 6419.6421.

[42] J. Marro and R. Dickman, Nonequilibrium Phase Transitions in Lattice Models, Cambridge University Press, Cambridge, 1985.

[43] B. Bollobás, Random graphs, Academic Press, New York, 1985.

[44] F. C. Santos and J. M. Pacheco, Scale-free networks provide a unifying framework for the emergence of cooperation, Physical Review Letters 95 (2005), 098104.

[45] F. C. Santos, J. F. Rodrigues and J. M. Pacheco, Graph topology plays a determinant role in the evolution of cooperation, Proc. R. Soc. London B 273 (2006), 50-55.

[46] A.-L. Barabási and R. Albert, Emergence of scaling in random networks, Science 286 (1999), 509-512.

[47] J. Gómez-Gardañes, M. Campillo, L. M. Floria and Y. Moreno, Dynamical organization of cooperation in complex topologies, Physical Review Letters 98 (2007), 108103.

[48] S. N. Dorogovtsev, J. F. F. Mendes and A. N. Samukhin, Size-dependent degree distribution of a scale-free growing network, Physical Review E 63 (2001), 052101.

[49] P. Erdôs and A. Rényi, On random graphs, Publ. Math. Debrecen 6 (1959), 290-297.

[50] D. J. Watts and S. H. Strogatz, Collective dynamics of 'small world' networks, Nature 393 (1998), 440-442.

[51] M. H. Vainstein and J. J. Arenzon, Disordered environments in spatial games, Physical Review E 64 (2001), 051905.

[52] B. J. Kim, A. Trusina, P. Holme, P. Minnhagen, J. S. Chung and M. Y. Choi, Dynamic instabilities induced by asymmetric influence: Prisoner's dilemma game in small-world networks, Physical Review E 66 (2002), 021907.

[53] P. Holme et al., Prisoner's dilemma in real-world acquintance networks: Spikes and quasiequilibria induced by the interplay between structure and dynamics, Physical Review E 68 (2003), 030901.

[54] G. Abramson and M. Kuperman, Social games in a social network, Physica D 208 (2005), $257-265$.

[55] Z.-X. Wu, X.-J. Xu and Y.-H. Wang, Prisoner's dilemma game with heterogeneous influental effect on regular small-world networks, Chinese Physical Letters 23 (2006), 531-534. 
[56] Z.-X. Wu et al., Evolutionary prisoner's dilemma game with dynamic preferential selection, Physical Review E 74 (2006), 021107.

[57] A. Szolnoki and G. Szabó, Cooperation enhanced by inhomogeneous activity of teaching for evolutionary prisoner's dilemma games, Europhysics Letters 77 (2007), 30004.

[58] A. Traulsen and M. A. Nowak, Evolution of cooperation by multilevel selection, Proc. Natl. Acad. Sci 103 (2006), 10952-10955.

[59] J. A. Fletcher and M. Zwick, The evolution of altruism: Game theory in multilevel selection and inclusive fitness, J. Theoretical Biology 245 (2007), 26-36.

[60] M. G. Zimmermann and V. M. Eguíluz, Cooperation, social networks, and the emergence of leadership in a prisoner's dilemma with adaptive local ineractions, Physical Review E 72 (2005), 056118.

[61] J. M. Pacheco, A. Traulsen and M. A. Nowak, Coevolution of strategy and complex networks with dynamical linking, Physical Review Letters 97 (2006), 258103.

[62] H. Ohtsuki, M. A. Nowak and J. M. Pacheco, Breaking the symmetry between interaction and replacement in evolutionary dynamics on graphs, Physical Review Letters 98 (2007), 108106.

[63] Z.-X. Wu and Y.-H. Wang, Cooperation enhanced by difference between interaction and learning neighborhoods for evolutionary spatial prisoner's dilemma games, Physical Review E 75 (2007), 041114. 
